\title{
WHY WE NEED TO “GRASP” OUR SURROUNDINGS: OBJECT AFFORDANCE AND PREHENSION IN ARCHITECTURE
}

\author{
Nikos SALINGAROS \\ University of Texas at San Antonio, Mathematics, One UTSA Circle, San Antonio, 78249 United States \\ E-mail:salingar@gmail.com
}

Received 04 April 2017; accepted 08 August 2017

\begin{abstract}
This paper argues for a basic need for graspable "handles" to be physically present, or merely suggested in our immediate environment. Yet two of the most characteristic elements of industrial-modernist architecture, plate-glass curtain walls and minimalist surfaces, fail to match this aspect of our body's biology. The absence of graspable components from the formal architecture of the $20^{\text {th }}$ Century leads to psychological disconnection on the part of the user, and could possibly be a cause for stress and anxiety. Physical built elements and designs suggestive of grasping arise in traditional and vernacular methods of construction as ubiquitous moldings, ornament, and trim in response to both human psychology and tectonics.
\end{abstract}

Keywords: architecture, complex analysis, composition tools, perception, the vision of the new system.

\section{Introduction}

A quiet revolution is underway, in which architects are beginning to prioritize human neurological responses in what they build (Robinson, Pallasmaa 2015; Sussman, Hollander 2015). How does the human organism react and relate to a building, space, surface, or structural detail? A recent collaborative effort between architects and scientists, with cross-fertilization among disciplines, is revealing important new findings that can be applied in buildings. This represents a paradigm shift towards evolutionary adaptation after decades during which design focused almost exclusively on form, abstract geometries, and industrial materials and construction techniques (Mehaffy, Salingaros 2015).

At the same time, we are discovering that traditional wisdom embedded in the built environment contains many of the design answers we now seek. Our ancestors who built towns and cities had an intuitive idea of which environments were more accommodating emotionally, and were therefore more healing (Alexander 1979; Alexander et al. 1977). People used their own direct senses as tools to evaluate the fitness of the built environment, and the most adaptive solutions survived a selection process of everyday use. Those older methods of choosing one design over another according to visceral response are now verified by present-day laboratory techniques (Salingaros 2017).

The sensory impact that our environment has on our nervous system and upon our body as a whole is the result of a complex mixture of signals from distinct sources, all of which affect us viscerally (Alexander et al. 1977; Pallasmaa 1996; Salingaros 2006). Our state of wellbeing is due in part to largely subconscious effects that environmental information triggers in our body, matching how our neurological system was optimized during our evolution for organismic survival. Instinctive responses to form, pattern, and surface fundamentally shape how we experience architecture. We can mask, but not change their effect on our body (Salingaros 2017).

\section{Different affordance mechanisms cooperate}

Innate neurophysiologic mechanisms inherited from our evolutionary past attach us to our environment instinctively (Robinson, Pallasmaa 2015; Sussman, Hollander 2015). Geometrical configurations adaptive to our body may be discovered and catalogued (Alexander et al. 1977). For example, "biophilia" defines a key mechanism within our neurological makeup of primary sensorial needs from the living environment (Kellert et al. 2008; Salingaros 2015). 
This mechanism connects us with biological forms, and with structures that mimic the special geometrical coherence of biological forms. Biophilia triggers unselfconscious connection to the healing geometry of our surroundings, if any is indeed present.

Biophilic design tries to apply rules of biological structure to the built environment. Two components of Biophilia are: (i) enabling intimate contact with life by including nature and plants in living environments; and (ii) coding the geometrical structure of biological forms into the actual fabric of the artificial built environment. The user then subconsciously connects to natural elements such as plants and surfaces built from wood or textured stone, etc., and to the mathematics of fractal scaling found in traditional and vernacular architectures. Fractal scaling is a key feature of plant structure and weathering in natural materials (Alexander 2001, 2002, 2004, 2005; Salingaros 2006, 2014, 2015).

Another, distinct connective mechanism independent of biophilia has to do with satisfying the sense of prehension, or grasping objects with our hands (Stuart 2013). We share this prehensile ability only with other primates. Prehension enables kinesthetic anchoring to the environment, an essential component of our need to be situated in the world. And "situatedness" - being embedded in the world through sensors - is one of the seven key properties of living structure (Salingaros, Masden 2015). The informational processes blend together and affect us through spatial perception mechanisms linked to our survival.

The present paper is devoted to outlining the prehensile aspect of object affordance. Our sensation of being situated in the environment clearly depends upon many distinct factors, one of which is the possibility of prehension. What all these mechanisms share is their common origin as key evolutionary adaptations for our ancestors. We have inherited those subconscious tools, and use them to judge and navigate vast stretches of artificial built environment that we now inhabit. Despite massive efforts at social engineering, basic human physiology cannot be changed, only suppressed, and that comes with damaging consequences for our wellbeing.

\section{Object affordance and our need to "grasp" nearby surfaces}

Experimental psychology reveals people's reaction to graspable objects in their immediate vicinity (Jeannerod et al. 1995; Portugali 1996). Neurological and hormonal signals prepare us mentally to grasp objects in our close environment that we perceive to fit our hand. Our brain identifies surroundings as be- ing accessible and touchable, a phenomenon called "object affordance". Our attention is drawn to clearly graspable shapes of between two and eight centimeters (about 1 to 3 inches). This prehensile effect is prompted by both the physical entity itself and its image in our eye and brain. Even an image of a graspable object affects our muscles directly, without our conscious mind making any explicit decision.

Our feeling of reassurance (situatedness) from being in a room depends in part on whether it offers real or apparent graspable "handles". These handles need not actually be grasped to provide the visceral benefit of their presence; they have only to be nearby and appear readily available for prehension. I propose several factors that, in the context of design, affect the intensity of this physiological and psychological object affordance effect positively or negatively. These are listed below as the Five architectural characteristics of object affordance:

(i) SIZE. A graspable item of a size to fit the average human hand helps us to perceive an environment as accommodating. It provides ready physical "support" should we ever need it. Common examples include handles, edges, trim, frames, moldings, as well as two-dimensional ornamental designs that offer us to hold them virtually.

(ii) SHAPE. The shape of physically-graspable structural elements in a room should invite comfortable hand contact (prehension), even if it never actually occurs. A shape is more likely to satisfy our interest if it offers smooth, rounded edges that will feel comfortable and good to the touch.

(iii) MATERIAL. Graspable elements or objects in a room that are made of transparent, random, or amorphous materials do not invite grasping. They are not defined strongly enough; they remain invisible to the mechanism of object affordance, hence the allure of prehension never occurs.

(iv) TEXTURE. Sharp, spiky, angular, rough, or otherwise visually unsettling objects, even if they are of the correct size and are situated within easy reach, signal possible pain or injury when grasped. Those repel instead of connecting positively. This sounds obvious, yet room surfaces and objects commonly found in rooms frequently violate it.

(v) DISTANCE. The object affordance effect is strongest for easily-reachable elements, and weakest for graspable elements that are situated outside our physical reach. It diminishes with distance. We connect best with accessible, touchable elements and surfaces showing graspable shapes and designs in the immediate vicinity. 
Object affordance acts subconsciously on people to situate them in space, hence in the world. Our motor neurons continuously perceive articulations and suggestive designs in our immediate surroundings (Garrido-Vásquez, Schubo 2014; McBride et al. 2012). Even if we don't notice those reactions on a conscious level, we still feel them as long as we are awake. The physiological state of our bodies - muscle tension and other neural activity triggered by object affordance - will consequently influence any action we take, including our reaction to just being there. A positive prehensile reaction enhances a person's wellbeing and performance, whereas a negative affordance set off by structural details that isolate us will influence all other actions negatively through superimposed anxiety and fatigue.

In his book The Thinking Hand, Juhani Pallasmaa (2009) discusses this topic from a philosophical point of view rather than that of experimental psychology. Coming from a respected architect and educator, his message could and should have influenced contemporary design in a positive, humanistic direction. Unfortunately it didn't, and we see examples from the work of architects who consistently eliminate built elements that might have invited "the thinking hand" to reach out for them. What happened is that the dictates of style impede intelligent ideas for improving the accommodating qualities of buildings.

\section{Scale dependence of space on small things}

Among the elements of a room, including its windows, doors, furniture, ornamental detail, and things like door knobs, handles, and window latches, the relationships of scale reveal a downward dependency: "The interdependence of scales is only one way: a higher scale requires all lower scales in order to function, but not vice versa." (Salingaros 2006: 75). This means that the composition and utility of larger architectural elements is influenced by all of the smallest pieces present, which either fit or fail to fit with the largest elements. Conceptually, this complex relationship is understood through smaller things literally upholding larger things.

Design components that correspond to bodily dimensions - our height, the length of our arms, the breadth of our hands, fingers, eyes, lips, nostrils, etc. in the range of 1 millimeter to 2 meters $(1 / 16$ inch to 6 feet) - play a major supportive role in how we use buildings and urban spaces. The existence and organization of ordered structure on these physical human scales shapes our experience of the whole, and this goes beyond thinking about them as optional details or ornament.
In design, no single scale should be valued above others, nor can it be sacrificed without understanding whether it serves a connective systemic - as opposed to an optional artistic - function (Alexander 2001, 2002, 2004, 2005). This sophisticated idea is not understood by prevailing architectural culture, since many of its standard design practices contradict it. Concentrating solely on the large-scale forms of a building or urban complex, as is often done nowadays, leads to negative adaptation. Without smaller and intermediate scales, geometrical coherence and situatedness are impossible.

The explanation for prehension comes from the theory of complex systems. In evaluating the fitness of a design, small and intermediate scales may not always be justifiable by aesthetic or even structural considerations, yet those scales are essential in adding systemic support to the whole. Object affordance that helps situatedness is actually essential for any larger spatial experience (Salingaros 2017).

A positive neurophysiologic interaction with our surroundings demands the full spectrum of human scales in architecture (Salingaros 2006, 2015). The smallest structural scales from $1 \mathrm{~mm}$ up to $2 \mathrm{~cm}(1 / 16$ to 1 inch) are very often present when we decide to (and can afford to) use natural materials. Sometimes, but not very often, the next higher scales from $2 \mathrm{~cm}$ to $8 \mathrm{~cm}$ (1 to 3 inches) are also included in natural grain and patterns. Yet we cannot assume that those scales will automatically arise in the materials. Materials do not normally show graspable dimensions (1 to 3 inches); therefore, to profit from object affordance, those scales need to be built and defined on purpose.

User wellbeing is linked to all the tectonic elements smaller in size than the human body cooperating to define an enveloping space in which one feels situated psychologically. Our brain perceives the small and intermediate size structural elements together as either being coherent (when we perceive a "whole"), or not (when we perceive randomness). Architects wishing to create the healing response triggered by geometrical coherence have to apply specific design techniques. Frames, trim, moldings, baseboards - all traditionally ornamental tectonic elements in a building that were eliminated in pursuing a minimalistic modernism are now encouraged to come back. Not as decoration, nor for aesthetic or stylistic reasons, but because they are needed to anchor the large-scale spatial form within our cognitive system.

\section{Minimalist environments are created not to adapt}

Oversimplified environments are intentionally bereft of graspable subdivisions and designs. Structurally 
necessary frames and supports in such designed spaces that could have satisfied real or virtual prehension are hidden. "Graspable" handles are generally missing, or in many cases made either too small or too large to grasp, as an intentionality of this peculiar design aesthetic. A minimalist approach to design frustrates the object affordance mechanism, diminishing or eliminating any prehensile connection with our surroundings.

Present-day architectural culture eliminates the human scales from design, which fits in with industrial-modernist top-down interventions, generic building, and urban renovation projects. Materials as used in industrial-modernism offer either no texture (e.g. glass and polished metal), or random surface patterns (e.g. concrete and bonded brick). The result is that there is no nested hierarchy of mathematical scales: the only scale present is defined at the size of the entire panel or wall. The characteristic scaled (fractal) substructure of natural materials, biological forms, and traditional-vernacular architecture is missing. Without scaling information, users of those spaces feel lost and anxious.

That minimalism is a purely ideological objective is evident, despite voluminous but misleading writings arguing for the necessity of this style in the modern world. Industrial materials have the characteristic of plasticity, and have been used in many occasions to define articulations at the intermediate and smaller scales. Metal panels can be cast or manufactured with ornament; concrete can be molded with patterns and its surface made friendly using paint or a ceramic aggregate, etc. The possibilities of creating nested substructure using industrial materials are endless. Yet existing built examples are condemned by architectural culture, so that students are either unaware of them, or are conditioned to shun the very idea.

\section{Curtain walls and machine precision do not help our wellbeing}

Note that most of the smaller components that are essential structural reinforcements are not decoration. Eliminating them for reasons of design style throws a considerable burden on precision required when using large tectonic elements exclusively. Furthermore, erasing the smaller architectural scales removes the possibility of useful adjustments on those scales (Alexander et al. 1977: Pattern 240). That drives up the cost, and forces us to rely upon large standard-size modules, which severely limits design freedom and adaptability. Small-scale trim is a far cheaper way of covering up imprecision in the larger tectonic components.

A building's construction budget is very often squandered on the abstract aesthetic goal of "machine precision", carefully controlling straight lines, joins, and edges to a very small tolerance, which does nothing towards the users' health and wellbeing. Ordinary people are not neurologically affected by machine precision (though many architects, because of their training, get very emotional over this). This false association of precision and design purity as being somehow redemptive has taken over architectural culture.

The older grilles or muntins between window panes, and the mullions between entire window units provide the crucial psychological attachment of prehension; not the transparent glass. Small window panes traditionally come with a "graspable" frame of exactly the right thickness to satisfy object affordance, and most traditional muntins would fit the hand nicely (Alexander et al. 1977: Pattern 239). Neurophysiology rules against the undifferentiated glass curtain wall. A large window made from plate glass with either minimal or no internal frame, nor any subdivisions, creates anxiety rather than a spatial experience that would situate our body via prehension.

Another instance of object affordance occurs in traditional bay windows (Alexander et al. 1977: Pattern 180). Here, a reassuring spatial environment partially envelops one or more persons while connecting visually to the outside. Object affordance acts on the scale of the entire body. Using the vocabulary of biophilia, successful bay windows combine the two factors "refuge" with "prospect" (Kellert et al. 2008; Salingaros 2015). Do not confuse glass curtain walls (which offer no information, hence no protective psychological boundary) with the solid structural frame/grid that defines the spatial and visual boundary of bay windows (which give the cognitive experience of prehension). They have opposite effects.

\section{Architectural experience depends upon layers of complexity}

Let us delve into the biological reasons for why prehension and object affordance affect us. Every complex system has different layers of complexity built onto a core foundation. This is evident in evolved organs such as the brain, in which basic neural modules identified as "primary" or "primitive" brains are nested inside our own more developed one (Sussman, Hollander 2015). Those evolutionary older portions are essential because our organism depends upon them for its basic life functions. The more evolutionary advanced layers that make us intelligent need those basic processing modules for automatic responses and regulatory functions.

DNA has a similarly nested complex structure, since our genetic code contains many pieces inherited from more primary or primitive life forms. A recent surprise (and blow to our ego) was to discover that those parts of our DNA that differ from our closest animal relatives, 
and which make us characteristically human - hence infinitely more advanced -, are relatively few.

In a direct analogy, we can identify primary elements of architectural form and space that exert the strongest influence on users. Those elements trigger an immediate, visceral response to built form. What we normally perceive as "architecture" is a complex ensemble of body reactions (Salingaros 2017). But many people make the mistake of restricting architectural experience to the surface layer of several cognitively-nested layers of a complex informational system; namely, the layer corresponding to "style". This reaction is learned from architectural culture. Less obvious yet primary (or primal) aspects of a building and space decide our predominant physiological and psychological responses; not style. The success of a building or an urban space depends on these innate elements more than on intellectual analysis of its structure.

Following the investigations of Christopher Alexander (2001, 2002, 2004, 2005), adaptive architecture relies upon the explicit organization of perceived complexity (Mehaffy, Salingaros 2015; Salingaros 2006, $2011,2014)$. The underlying assumption is that our neurophysiology developed for the purpose of analyzing information automatically. A science-based theoretical model helps to explain how we react to different environments. We pick up useful pieces of complex information from our surroundings subconsciously. Other, separate layers of our cognitive apparatus synthesize this information to compute an integrated result. Our body then acts and reacts according to this internal cue.

Traditional building forms adapted to our neurophysiology so that we felt more at ease, as long as we built using complex emotional feedback as a guide. Such buildings depend upon all their individual internal elements, spaces, and surfaces - which traditionally followed criteria that guarantee a positive emotional connection -cooperating cognitively. Adaptive buildings optimize the tectonic system's geometrical coherence, and consequently enhance their ability to accommodate human life in all its biological complexity. This is the reason why traditional buildings boost our wellbeing.

\section{Can we save money by eliminating information?}

We are constantly feeding on environmental information, but only if that information belongs to a special category that we evolved to handle. Otherwise, information (or lack of it) confuses us and makes us anxious. If highly sophisticated environmental complexity is either absent, or it overwhelms us, this cre- ates anxiety because we cannot process its embedded information to help us live. Anxiety leads to a fightor-flight response, which ethical architects should avoid at all costs (although, perversely, some of them actually seek to induce it on their buildings' users). Investigating how complexity can be organized geometrically gives us a tool with which to understand why our neurophysiology is set up to respond the way it does (Salingaros 2006, 2011, 2014).

The commonly-heard justification linking design minimalism to utility turns out to be unfounded. Contrary to popular thinking, removing information does not make a structure more useful by making it more generic (Brand 1995). Information and substructure should be removed from a design only if those are clearly responsible for geometrical dissonance that destroys psychological situatedness. Stripping down a space actually reduces its utility for any use requiring human participation, leaving us with an industrial shed. It loses its human qualities. We see this poverty in environments that have been oversimplified through misguided top-down interventions (Salingaros 2011).

The cost-saving agenda of industrial standardization and modularization never benefits the human user. A room of some standard size, with fixed ceiling height, window sizes and placements will not "fit" most situations emotionally, because those require distinct needs for entirely distinct users, conditions, connections, etc. Neither can an architect re-use an identical minimal module in designing spaces for different uses, different climates, different societies, and different placement within a building in relation to all the other rooms, paths, and spaces. Millions of sensory and cognitive cues have to be satisfied when designing adaptively, which together define a psychologically healthy environment. This reasoning rules out generic International Style design (triggering opposition from those who have bought into its supporting ideology).

\section{Including all of our other senses}

In The Eyes of the Skin, Juhani Pallasmaa (1996) discusses the non-visual components of sensory experiences interpreting our environment, which contribute to how structures affect our body viscerally. Pallasmaa tried to break out of the exclusive focus on visual style that drove architecture in the $20^{\text {th }}$ Century. While his book is an assigned reading in architecture schools, it does not seem to have the hoped-for impact either in studio, or in practice. Architects love to refer to it, yet invariably, their designs fail to embody the qualities it describes! Instead of training architects to be sensitive to non-visual environmental interactions that either trigger or reduce anxiety, conventional design 
pedagogy ignores them, and focuses instead on formal approaches and visual novelty.

Environmental stimuli are constantly being interpreted by our sensory system, generating physiological responses felt within our entire body. This effect depends upon superimposed contributions coming from all of our senses. Those include - but are not limited to - sight, hearing, smell, balance, touch, invisible electromagnetic radiation (for example, infrared heat exchanged from hot or cold surfaces, and frequencies that our body experiences directly), and a kinesthetic awareness of our surrounding space. Moreover, contributions from distinct mechanisms act on different scales and at different ranges. The complex character of these interactions changes with the physical distance between the human body and a structure or surface.

The ambient information field is akin to a force field that ties us to our surroundings (even though there is no physical exchange taking place). But "image-based design" ignores mechanisms responsible for situatedness. Details that define a welcoming environment are either missing, or are juxtaposed incoherently in many of today's buildings. Architects who focus on the purity of abstract geometry ignore the ensemble of complex forces acting on our body. The result leads to the opposite of situatedness: a sense of "placelessness", and even anxiety.

An abstract design that looked fine in a rendering, but which failed to evaluate -and adjust for - all predictable human sensory reactions could turn out to be a threatening and oppressive environment when built. Users will avoid those architectural spaces, or force themselves to use them while fighting increased stress levels. Apparently benign design decisions based on abstractions can trigger negative physiological responses in the user. This comes from not thinking about the consequences, or worse, having being falsely taught that there are no consequences. Architects stick religiously to the primacy of "image-based design".

The countless complex interactions that combine to generate a visceral signal determine a comparatively simple set of instinctive behaviors for the user. Our body tells us what to do without thinking. This result is more basic than either psychology or medicine, and underlies the physical experience of architecture. It cannot be overridden by formal design. A space designed for a predetermined function and use could be more suitable for a totally different behavior; or it could be dysfunctional, because our body is reacting viscerally to that space's hostile geometry, surfaces, details, and complexity (or lack of it) in an unexpected way. Those architectural mistakes have been ignored for too long: it's time to apply science to fix them.

\section{Past and future studies}

The topic of affordances goes back to $19^{\text {th }}$ Century theories of "empathy aesthetics", especially the work of Heinrich Wölfflin (Wagner, Blower 2014). A considerable and interesting literature on how the human body reacts with and is situated in the environment was swept away when architectural culture adopted the Bauhaus and International Style building ideology. Later, in the field of psychology, James J. Gibson formulated his theory of affordances (Gibson 1979). Gibson's work initiated a research program that continues to this day, but which has yet to influence architectural design. I have already mentioned the pioneering work of Pallasmaa in trying to bring those concepts into architecture (Pallasmaa 1996, 2009).

The situation is changing because new experimental research in neuroscience is discovering how we react to different environments (Fritz, Palti 2017; Robinson, Pallasmaa 2015; Shemesh et al. 2016; Sussman, Hollander 2015). Direct measurements of our brain and body's strong reactions to environmental cues can no longer be ignored by architectural culture, especially when it becomes clear that human health and wellbeing are at stake. Even so, architects have so far been able to avoid human adaptive design because the profession is still driven by fashion, inertia, money, and resistance to change.

Instead of engaging in polemics with an antiquated and dogmatic system, the way forward is through more scientific research. Several groups are pursuing clinical studies that differentiate between harmful and healing features of our environment. There is much confusion so far, principally because of hidden bias within architectural culture. Architects desperately wish to save their cult heroes, even when those buildings are psychologically unhealthy for their users. Abusing their supposed authority, architects mislead research scientists by telling them what is "great" architecture, before measuring psychological affordance (Salingaros 2017).

\section{Conclusions}

A new, adaptive approach to architecture and design pays close attention to human evolution. Human beings respond to spaces, surfaces, detail, and ornament viscerally, which determines how a built structure will actually be used independently of whatever the architect intended. Yet as long as the design has followed basic adaptive principles alluded to here, a user should feel situated in that space. Design components joined together coherently communicate a strong subconscious message. Sometimes, surprising emergent phenomena could enhance the users' experience of the built structure. In the best of cases, the building, or portions of it, evoke a "sense of belonging" that situates 
users. The built environment thus acquires welcoming properties. In exceptional cases, moreover, this perception could translate into a "sense of wonder", such as occurs in the great religious buildings of the past.

Finally, the interacting system of building-plususer is not only a system in space, but also one in time. Geometrical components interact with users on distinct spatial scales, but we should also design for the different and changing movements of people, users of different characteristic time periods, for the changing time of day, etc. All those temporal scales need to interact in a coherent manner, coordinated rather than restricted and frustrated by some "designed" form. Whenever emergent design is successful, the complex temporal system will interact seamlessly with the complex spatial system (while treated independently for practical reasons during the design process) as one space-time system. We now possess the tools to create adaptive, healing environments that are better for our health. Those take us away from the practice of "architecture as image".

\section{References}

Alexander, C. 1979. The timeless way of building. New York: Oxford University Press.

Alexander, C. 2001. The nature of order: the phenomenon of life. Berkeley, California: Center for Environmental Structure.

Alexander, C. 2002. The nature of order: the process of creating life. Berkeley, California: Center for Environmental Structure.

Alexander, C. 2005. The nature of order: a vision of a living world. Berkeley, California: Center for Environmental Structure.

Alexander, C. 2004. The nature of order: the luminous ground. Berkeley, California: Center for Environmental Structure.

Alexander, C.; Ishikawa, S.; Silverstein, M.; Jacobson, M.; Fiksdahl-King, I.; Angel, S. 1977. A pattern language. New York: Oxford University Press.

Brand, S. 1995. How buildings learn. New York: Penguin Books.

Fritz, A.; Palti, I. (Eds.) 2017. Conscious cities: bridging neuroscience, architecture, and technology. Anthology No. 2., in the $2^{\text {nd }}$ conscious conference, $3^{\text {rd }}$ May 2017, London [online], [cited 1 September 2017]. Available from Internet: ccities.org/publications/conscious-cities-bridging-neuroscience-architecture-technology/

Garrido-Vásquez, P.; Schubo, A. 2014. Modulation of visual attention by object affordance, Frontiers in Psychology, 6 February 2014 [online], [cited 1 September 2017]. Available from Internet: journal.frontiersin.org/article/10.3389/ fpsyg.2014.00059/abstract

Gibson, J. J. 1979. The theory of affordances, Chapter 8, in The Ecological Approach To Visual Perception. Hillsdale, New Jersey: Lawrence Erlbaum.

Jeannerod, M.; Arbib, M. A.; Rizzolatti, G.; Sakata, H. 1995. Grasping objects: the cortical mechanisms of visuomotor transformation, Trends in Neurosciences 18: 314-320. https://doi.org/10.1016/0166-2236(95)93921-J

Kellert, S. R.; Heerwagen, J.; Mador, M. (Eds.) 2008. Biophilic design: the theory, science and practice of bringing buildings to life. New York: John Wiley.
McBride, J.; Boy, F.; Husain, M.; Sumner, P. 2012. Automatic motor activation in the executive control of action, Frontiers in Human Neuroscience, 24 April 2012 [online], [cited 01 September 2017]. Available from Internet: journal.frontiersin.org/article/10.3389/fnhum.2012.00082/abstract

Mehaffy, M. W.; Salingaros, N. A. 2015. Design for a living planet: settlement, science, and the human future. Portland: Sustasis Press.

Pallasmaa, J. 1996. The eyes of the skin. New York: John Wiley.

Pallasmaa, J. 2009. The thinking hand. Chichester, UK: John Wiley.

Portugali, J. (Ed.) 1996. The construction of cognitive maps. Dordrecht, Holland: Kluwer Academic. https://doi.org/10.1007/978-0-585-33485-1

Robinson, S.; Pallasmaa, J. (Eds.) 2015. Mind in architecture. Cambridge, Massachusetts: MIT Press.

Salingaros, N. A. 2006. A theory of architecture. Solingen, Germany: Umbau-Verlag.

Salingaros, N. A. 2011. Why monotonous repetition is unsatisfying, Meandering Through Mathematics, 2 September 2011 [online], [cited 01 September 2017]. Available from Internet: meandering-through-mathematics.blogspot.com/2011/09/ why-monotonous-repetition-is.html

Salingaros, N. A. 2014. Complexity in architecture and design, Oz Journal 36(May 2014): 180-25. https://doi.org/10.4148/2378-5853.1527

Salingaros, N. A. 2015. Biophilia and healing environments. Amherst, Massachusetts: Off the Common Books.

Salingaros, N. A. 2017. How neuroscience can generate a healthier architecture, Conscious Cities Journal 3(August 2017) [online], [cited 01 September 2017]. Available from Internet: ccities.org/publications/conscious-cities-journal-no-3/

Salingaros, N. A.; Masden, K. G. 2015. Architecture: Biological Form and Artificial Intelligence, updated version of older article with new sections added, $A+U$, Part 1 in 540(September 2015): 130-135, Part 2 in 541(October 2015): 152-155, Part 3 in 542(November 2015): 209-212, Part 4 in 543(December 2015): 124-129.

Shemesh, A.; Talmon, R.; Karp, O.; Amir, I.; Bar, M.; Grobman, Y. J. 2016. Affective response to architecture - investigating human reaction to spaces with different geometry, Architectural Science Review 60(2): 116-125. https://doi.org/10.1080/00038628.2016.1266597

Stuart, S. A. 2013. Privileging exploratory hands: prehension, apprehension, comprehension, Chapter 14, in Z. Radman (Ed.). The Hand, an organ of the mind [online], [cited 01 September 2017]. Cambridge, Massachusetts: MIT Press, 329-347. Available from Internet: eprints.gla. ac.uk/60040/1/60040.pdf

Sussman, A.; Hollander, J. B. 2015. Cognitive architecture. New York: Routledge.

Wagner, K.; Blower, J. 2014. Animating architecture: empathy and architectonic space, Art in Translation 6(4): 399-436. https://doi.org/10.1080/17561310.2014.11425537

\section{NIKOS SALINGAROS}

Is an internationally-known urbanist and architectural theorist. The author of seven books and numerous scientific publications, he is Professor of Mathematics at the University of Texas at San Antonio. 